\title{
Patient Satisfaction with Virtual-Based Prenatal Care: Implications after the COVID-19 Pandemic
}

\author{
Cindy H. Liu ${ }^{1,2,3}$ (D) . Deepika Goyal ${ }^{4} \cdot$ Leena Mittal ${ }^{2,3} \cdot$ Carmina Erdei $^{1,3}$ \\ Accepted: 8 August 2021 / Published online: 19 August 2021 \\ (c) The Author(s), under exclusive licence to Springer Science+Business Media, LLC, part of Springer Nature 2021
}

\begin{abstract}
Objective The objective of this study was to identify factors related to satisfaction with virtual visits during pregnancy in an effort to prioritize intervention targets for pregnant women during the COVID-19 pandemic.

Methods The study relied on data obtained from pregnant women $(N=416)$ who participated in the Perinatal Experiences and COVID-19 Effects (PEACE) Study from May 21 to November 22, 2020. Using a cross-sectional design, this study examined factors including COVID-19 related experiences and prenatal care changes in association with patient satisfaction of virtual prenatal care.

Results Overall, women reported being very or extremely satisfied (27.9\%) or moderately satisfied (43.5\%) with their virtual prenatal experiences, however, $89.9 \%$ indicated a preference for in-person care under non-pandemic conditions. Those who completed the survey further into the pandemic were less satisfied with virtual prenatal care $(\beta=-0.127, p<0.01)$. After accounting for this and other sociodemographic characteristics, COVID-19 pregnancy worries $(\beta=-0.226, p<0.001)$ and the number of prenatal care changes due to the pandemic $(\beta=-0.137, p<0.01)$ were associated with lower satisfaction.

Conclusion Our findings demonstrate general satisfaction with virtual visits among pregnant women in this study although in general women would prefer in-person care if it weren't for a pandemic. Women worried about the impact of pandemic on their pregnancy, as well as those experiencing transitions in their prenatal care may need more information and reassurance. Additional studies are needed to understand the unmet needs through virtual care compared to in-person care.
\end{abstract}

Keywords Telehealth $\cdot$ Pregnancy $\cdot$ Mental health $\cdot$ Anxiety $\cdot$ Obstetric

\section{Significance}

Little is known regarding patient satisfaction of virtual prenatal care during the COVID-19 pandemic. Existing studies on patient satisfaction of virtual care have not accounted for current mental health and other psychosocial factors related to the COVID-19 pandemic.

Cindy H. Liu

chliu@bwh.harvard.edu

1 Department of Newborn Medicine, Brigham and Women's Hospital, Boston, MA, USA

2 Department of Psychiatry, Brigham and Women's Hospital, Boston, MA, USA

3 Harvard Medical School, Boston, MA, USA

4 The Valley Foundation School of Nursing, San Jose State University, San Jose, CA, USA
Women worried about the impact of the pandemic on their pregnancy and those experiencing more transitions in their prenatal care may need more information regarding their care. Despite the reported general satisfaction of virtual prenatal care, the large majority of women had a preference for in-person visits indicating the need for better understanding of the unmet needs through virtual prenatal care.

\section{Introduction}

Pregnant women are classified as a vulnerable population, with COVID-19 increasing their risks for hospitalization, ventilator assistance, and preterm labor (National Center for Health Statistics, 2020). To limit prenatal COVID19 exposure, the American College of Obstetricians and Gynecologists and the Center for Disease Control and Prevention suggested including some virtual visits in place of routine prenatal care (Lowery et al., 2020; National Center 
for Health Statistics, 2020). Indeed, prenatal care models with telehealth appointments have been implemented (Aziz et al., 2020; Fryer et al., 2020; Peahl et al., 2020a; Reforma et al., 2020; Zork et al., 2020). However, given the rapid shift from in-person care to virtual visits, very little is known regarding patient satisfaction of virtual prenatal care during the pandemic.

Telehealth or virtual care are modalities that comprise of synchronous video visits or audio-only visits, asynchronous communication, and remote patient monitoring (Lowery et al., 2020). Although a promising direction for care, the handful of studies on patient satisfaction with virtual prenatal care during the pandemic have shown mixed findings. For example, in a study of audio-only virtual prenatal visits in Dallas, Texas $(N=283)$, the majority of women described their experience as good or very good and preferred a combination of in-person and virtual visits for prenatal care (Holcomb et al., 2020). Another study compared satisfaction between in-person and telehealth prenatal care among a diverse but low risk sample of 104 women from East Harlem (Futterman et al., 2020). Women had statistically significant lower rates of satisfaction from televisits compared to inperson care. Among 91 high risk obstetric patients across a New York health care system who had telehealth visits, $86.9 \%$ were satisfied with the care they received and $78.3 \%$ indicated that they would recommend telehealth visits to others (Jeganathan et al., 2020).

Patient satisfaction is an important outcome when evaluating the success of telehealth approaches (Kruse et al., 2017). Yet, the existing studies on patient satisfaction of virtual care have not accounted for factors that may be associated with satisfaction such as current mental health symptoms or psychosocial factors related to the COVID19 pandemic. For instance, women with anxiety, may be concerned about COVID-19 exposure and may self-impose stricter social distancing practices (Imlach et al., 2020); they may show greater satisfaction or preference with virtual prenatal care. On the other hand, those with anxiety could also be less satisfied with their virtual prenatal care experience, as fetal assessments such as heart rate and abdominal measurements cannot be easily assessed virtually. As well, worry about the impact of the COVID-19 pandemic, as it relates to getting health care or support may lead a patient to desire in-person care although some may prefer the convenience of virtual visits. Altogether, the extent to which prior mental health conditions and current COVID-19-related concerns might affect women's satisfaction of virtual prenatal care is not well understood.

The purpose of this study was to understand the factors involved in virtual prenatal care satisfaction among women pregnant during the COVID-19 pandemic. Besides sociodemographic variables, we considered potential predictors such as current mental health symptoms. Importantly, we examined the extent to which reported COVID-19-related emotional experiences (pregnancy worries, grief, and health worries) and the extent to which prenatal changes that took place due to the COVID-19 pandemic accounted for reported satisfaction levels. Preferences for virtual care under nonpandemic conditions were also examined.

\section{Methods}

\section{Participants}

The data was drawn from the Perinatal Experiences and COVID-19 Effects (PEACE) study (www.peacestudy2020. com), an online survey administered to U.S. pregnant and postpartum women during the COVID-19 pandemic. The eligibility and recruitment of the study has been described elsewhere (Liu et al., 2021a). Briefly, the present analysis includes those over the age of 18 years, and in their second or third trimester of pregnancy. A snowball sampling approach relying on social media and email listservs was used for recruitment. The study procedures were approved by the Mass General Brigham Institutional Review Board. The cross-sectional analyses for this particular study included women who responded to our survey from May 21, 2020 to November 22, 2020, who indicated yes to the question "Did you receive virtual prenatal care since the onset of the COVID-19 pandemic?" $(N=416)$.

\section{Measures}

\section{Predictors}

To assess current depressive symptoms, we relied on the Center for Epidemiologic Studies-Depression (CES-D; Radloff, 1977), a 20-item self-report measure that assesses the frequency of symptoms experienced over the past week. The total possible range for scores is 0 -to-60. The sum score was used, with higher scores reflecting greater depressive symptoms was used for analyses (Lewinsohn et al., 1997).

Participants completed the generalized anxiety disorder scale (GAD-7; Spitzer et al., 2006), a 7-item self-report measure that captures participants' frequency of current anxiety symptoms from the past two weeks. The possible range for scores is 0 -to-21. The analyses used sum scores, with higher scores indicating elevated anxiety (Plummer et al., 2016).

To capture current PTSD symptoms, we used the PTSD Checklist-Civilian Version (PCL-C; Weathers et al., 1993), a standardized 17 -item self-report rating scale that measures the extent to which participants' have been bothered by problems and experiences in response to stressful life events over the past month. The possible range for 
scores is 17-to-85. As with the other symptom measures, sum scores were used for analyses, with higher scores reflecting greater PTSD symptoms.

To obtain feelings of worry specific to pregnancy during the COVID-19 pandemic, an 8-item measure was used. These items included a list of worry/stress experiences such as "I don't have a way to get to the hospital if I/my baby becomes sick and I need to see a doctor" or "not receiving adequate prenatal care due to COVID-19." Participants were asked to use a five-point scale with $1=n o t$ worried/stressed at all and 5=very worried/stressed. We conducted an exploratory factor analysis with the 8 items prior to its use, where we applied a principal-axis factor analysis with varimax rotation to yield factors that had eigenvalue greater than 1 . From this, two factors with an eigenvalue of 3.53 and 1.06 were obtained. These factors were evaluated based on the item factor loadings $(>0.30)$ and whether the items within each factor made conceptual sense. As such, one factor consisted of five items with factor loadings ranging from 0.41 to 0.72 , and a second factor consisted of three items with factor loadings ranging from 0.54 and 0.66 , with factors accounting for 22.5 and $20.3 \%$ of the total variance, respectively. For our analysis, the measure was scored by taking the sum of all 8 items. A Cronbach's alpha coefficient of 0.81 was obtained for this scale, indicating good reliability. The sum score was calculated and used for analyses with higher scores reflecting greater worry. As expected, pregnancy worry scores were associated with generalized anxiety symptoms in pregnant and postpartum women $(r=0.329, p<0.001)$.

We used a 7-item measure to ascertain experiences of grief related to the COVID-19 pandemic, with three items that inquired about missing out on celebrating major life events, loss of resources, and loss of support of family and friends because of social distancing, and three items adapted from the inventory of complicated grief (Prigerson et al., 1995). One item focused on the perinatal experience assessed sad feelings due to being unable to celebrate the pregnancy and/or birth of their child with loved ones because of the COVID-19 pandemic. Participants were asked to rate each of the items on a five-point scale with $1=$ strongly disagree and $5=$ strongly agree. The measure had a Cronbach's alpha coefficient of 0.78, demonstrating good reliability. Higher summed scores reflected higher levels of grief.

To assess worry specifically related to the effect of COVID-19 on health, four items from the Coronavirus Health Impact Survey (CRISIS) were used (Merikangas et al., 2020). Participants rated their concerns regarding becoming infected, their friends and family becoming infected, and their physical and mental health being influenced by the COVID-19. A five-point scale of $1=$ not at all and 5=extremely. The measure had a Cronbach's alpha coefficient of 0.78 , showing good reliability. Higher summed scores indicated higher levels of health worries.

Respondents were asked to indicate prenatal care changes as a result of the COVID-19 outbreak. The full list of items is presented in Table 3. The sum of endorsed items was calculated and used as a continuous predictor. Items were recoded into $0,1-2,3-4$, and $\geq 5$ changes for descriptive purposes.

\section{Outcomes}

Participants were asked to rate how satisfied they were with their virtual prenatal care experience using a 5-point scale, with $1=$ not at all and $5=$ extremely.

Participants were asked how much they would prefer virtual prenatal care in between required in-person visits (e.g., for ultrasounds) under non-pandemic conditions. Options included "I would very much prefer in-person prenatal care" and "I would somewhat prefer in-person prenatal care" which were collapsed into "Prefer in-person prenatal care." "I would very much prefer virtual prenatal care" and "I would somewhat prefer virtual prenatal care" were collapsed into "Prefer virtual prenatal care." "I have no preference" was not recoded.

\section{Covariates}

Maternal characteristics including age, education, and household income were obtained as well as whether this was the participants first pregnancy and whether they were in their second or third trimester of pregnancy. The number of days from the date when COVID-19 was declared as a pandemic (March 13, 2020) to each participant's survey start date was calculated. Given possible changes in the experience of the pandemic over time, this variable was included as a covariate.

\section{Data Analytic Plan}

The means and frequencies of the sample characteristics (sociodemographic variables, pandemic duration at the time of the survey completion), and key variables including mental health symptoms, COVID-19 related experiences, virtual prenatal care satisfaction, and prenatal care changes were calculated for descriptive purposes. We conducted hierarchical multiple regressions to examine predictors of virtual prenatal care satisfaction among pregnant women. Covariates and predictor variables were entered sequentially into the model to predict satisfaction scores. Covariates included sociodemographic characteristics (Block 1). Predictors were included as blocks with the second block including current mental health symptoms (depression, generalized anxiety, PTSD; Block 2), which was followed by COVID-19 
experiences (pregnancy worries, grief, and health worries; Block 3). We then entered in the number of prenatal care changes (Block 4) in predicting satisfaction scores. Finally, a chi-square analysis was performed to determine the association between virtual vs. in-person prenatal care preferences under non-pandemic conditions and patient satisfaction of the virtual prenatal care received.

\section{Results}

The sample characteristics from our analytic sample are presented in Table 1. Participants were on average, 32.7 years of age, largely White (92.5\%) and college educated (93.1\%), with $43.3 \%$ reporting household incomes of more than $\$ 150,000$. This was the first pregnancy for slightly less than half (46.9\%) of our sample, with $38.0 \%$ in their second trimester and $62.0 \%$ in their third trimester of pregnancy. Respondents on average completed the survey 120 days ( 4 months) after the start of the pandemic.

We refer the reader to Table 2 for the descriptives on predictors and outcomes. We note that $27.9 \%$ indicated being

Table 1 Sample characteristics of pregnant women from the PEACE study, data collected between May 21 to November 22, 2020

\begin{tabular}{ll}
\hline Predictors & Means (SD or \%) \\
\hline Maternal age (years) & $32.74(3.82)$ \\
Maternal education & \\
Less than college & $7.0 \%$ \\
College & $31.3 \%$ \\
Masters & $41.6 \%$ \\
Doctorate & $20.2 \%$ \\
Household income (USD/year) & \\
$<\$ 74,999$ & $12.3 \%$ \\
\$75,000-149,999 & $44.4 \%$ \\
\$150,000-224,999 & $27.3 \%$ \\
$>\$ 225,000$ & $16.0 \%$ \\
Maternal race & \\
White & $92.5 \%$ \\
Black or African American & $0.7 \%$ \\
Hispanic or Latino & $3.4 \%$ \\
Asian and Pacific Islander & $3.4 \%$ \\
First pregnancy & \\
No & $53.1 \%$ \\
Yes & $46.9 \%$ \\
Pregnancy trimester &
\end{tabular}

$N=416$
Table 2 Key variable characteristics of postpartum women from the PEACE study, data collected between May 21 to November 22, 2020

\begin{tabular}{ll}
\hline Predictors & Means or \% (SD, range) \\
\hline Current mental health symptoms & \\
Depression (CES-D) & $14.51(8.99,0-53)$ \\
Generalized anxiety (GAD-7) & $6.14(4.78,0-21)$ \\
PTSD (PCL-C) & $28.95(9.69,17-69)$ \\
COVID-19 experiences & \\
COVID-19 pregnancy worries & $20.55(6.32,6-37)$ \\
COVID-19 grief & $21.03(4.73,7-33)$ \\
COVID-19 health worries & $12.08(3.66,4-20)$ \\
Virtual prenatal care satisfaction & \\
Not at all & $9.6 \%$ \\
Slightly & $19.0 \%$ \\
Moderately & $43.5 \%$ \\
Very & $23.6 \%$ \\
Extremely & $4.3 \%$ \\
\hline
\end{tabular}

$N=416$

"very" or "extremely" satisfied with their virtual prenatal care experience, with a plurality of responses indicating being "moderately" satisfied with their virtual prenatal care experience.

Table 3 presents the rates of specific prenatal care changes that took place as a result of the COVID-19 pandemic. The

Table 3 Rates of reported prenatal care changes due to the COVID19 pandemic among pregnant women, from the PEACE study, data collected between May 21 to November 22, 2020

\begin{tabular}{ll}
\hline Prenatal care changes by item & Rates (\%) \\
\hline Transition from in-person prenatal visits to virtual visits & 81.3 \\
Changed format of prenatal care (i.e. no group classes) & 70.4 \\
Cancellation of or reduction in frequency of prenatal & 64.7 \\
$\quad$ visit(s) & \\
Cancellation of hospital tours & 59.6 \\
Change in prenatal health care provider(s) & 11.5 \\
Change in selected hospital or birthing center & 5.0 \\
Changes in schedule for planned C-section or labor & 2.9 \\
induction & \\
Changed from planned vaginal birth to induction or & 1.2 \\
C-section & \\
Changed from plan for hospital delivery to a home birth & 1.2 \\
Changed from planned home birth to a hospital birth & 0 \\
Total prenatal care changes & \\
None & 3.8 \\
1-2 & 30.8 \\
3-4 & 58.2 \\
$\geq 5$ & 7.2 \\
\hline
\end{tabular}

$N=416$ 
majority of women reported transitioning from in-person to primarily virtual during their pregnancy $(81.3 \%)$, having

Table 4 Multiple regression predicting virtual prenatal care satisfaction among pregnant women during the COVID-19 pandemic, based on sociodemographic variables, mental health history and symptoms, COVID-19-related experiences, and reported number of prenatal care changes

\begin{tabular}{|c|c|c|c|}
\hline \multirow[t]{2}{*}{$\begin{array}{l}\text { Blocks of variables entered in three } \\
\text { steps }\end{array}$} & \multicolumn{3}{|c|}{$\begin{array}{l}\text { Virtual prenatal care satisfac- } \\
\text { tion }\end{array}$} \\
\hline & $\bar{\beta}$ & $R^{2}$ & $\Delta R^{2}$ \\
\hline 1. Covariates & & 0.023 & $0.046^{*}$ \\
\hline Maternal age & 0.076 & & \\
\hline \multicolumn{4}{|c|}{ Maternal education $($ reference $=$ less than college) } \\
\hline College & -0.08 & & \\
\hline Masters & -0.025 & & \\
\hline Doctorate & 0.047 & & \\
\hline \multicolumn{4}{|c|}{ Household income (reference $\leq \$ 74,999$ ) } \\
\hline$\$ 75,000-149,999$ & 0.020 & & \\
\hline$\$ 150,000-224,999$ & -0.013 & & \\
\hline$>\$ 225,000$ & -0.014 & & \\
\hline First pregnancy $($ reference $=$ no $)$ & 0.065 & & \\
\hline Pandemic duration & $-0.127 * *$ & & \\
\hline 2. Current mental health symptoms & & 0.064 & $0.047 * * *$ \\
\hline Depression & -0.028 & & \\
\hline Generalized anxiety & $-0.145^{\dagger}$ & & \\
\hline PTSD & 0.020 & & \\
\hline 3. COVID-19 Experiences & & 0.100 & $0.042 * * *$ \\
\hline COVID-19 pregnancy worries & $-0.226 * * *$ & & \\
\hline COVID-19 grief & -0.001 & & \\
\hline COVID-19 health worries & 0.077 & & \\
\hline $\begin{array}{l}\text { 4. Prenatal care changes due to } \\
\text { COVID-19 }\end{array}$ & & 0.113 & $0.015 * *$ \\
\hline Reported Number of changes & $-0.137 * *$ & & \\
\hline
\end{tabular}

Model does not include maternal race due to multicollinearity

$N=416$

${ }^{\dagger} p<0.1$

$* p<0.05$

$* * p<0.01$

$* * * p<0.001$ the format of their prenatal care changed (70.4\%), having cancellations or reductions in frequency of prenatal visits (64.7\%), and having their hospital tours cancelled (59.6\%). Almost two-thirds of women (65.4\%) reported at least three changes to their prenatal care as a result of the pandemic.

Table 4 refers to the associations observed between sociodemographic variables, current mental health symptoms, and COVID-19 experiences. Those who completed the survey later tended to be less satisfied with their virtual prenatal care experiences $(\beta=-0.127, p<0.01)$. We note a statistical trend with generalized anxiety symptoms associated with lower satisfaction with virtual prenatal care $(\beta=-0.145$, $p<0.10)$. After accounting for sociodemographic characteristics, and current mental health symptoms, women who scored higher on COVID-19 pregnancy worries were more likely to report lower satisfaction of virtual prenatal care $(\beta=-0.226, p<0.001)$. Finally, the higher number of reported prenatal care changes due to the COVID-19 pandemic was associated with lower virtual prenatal care satisfaction $(\beta=-0.137, p<0.01)$.

As shown in Table 5, the large majority of women preferred in-person prenatal care under non-pandemic conditions. When examining the preference for in-person versus virtual prenatal care, $89.9 \%$ of the total women with available data $(N=386)$ indicated a preference for in-person care, with $10.1 \%(n=39)$ indicating a preference for virtual care. Among them were women who were very or extremely satisfied (20.7\%), moderately satisfied (47.0\%), and not at all or slightly satisfied (32.3\%) with their virtual prenatal care experience. The low cell size of those who reported "no preference" prevented us from the chi-square analysis and thus these responses were removed. The rates among satisfaction by preference were significantly different $\left[\chi^{2}\right.$ $(2)=24.3, p<0.001$ ], exemplified with $56.4 \%$ of the women with a preference for virtual care reporting being very or extremely satisfied with their virtual prenatal care experience versus $20.7 \%$ of women who preferred in-person care reporting being very or extremely satisfied with their virtual prenatal care experience.

We also conducted a sub-analysis to examine whether these rates differed for first-time mothers and mothers who
Table 5 Rates of satisfaction of virtual prenatal care experiences by preference for virtual or in-person care under nonpandemic conditions among pregnant women, data collected between May 21 to November 22, 2020 from wave I of the PEACE study

\begin{tabular}{lll}
\hline $\begin{array}{l}\text { How satisfied are you with your virtual } \\
\text { prenatal care experience? }\end{array}$ & $\begin{array}{l}\text { "If we were not in a pandemic, how much would you prefer } \\
\text { virtual prenatal care in between required in-person visits (e.g. } \\
\text { for ultrasounds)? }\end{array}$ \\
\cline { 2 - 3 } \cline { 2 - 2 } $\begin{array}{ll}\text { Somewhat or very much prefer } \\
\text { virtual }(\mathrm{n}=39)(\%)\end{array}$ & $\begin{array}{l}\text { Somewhat or very much } \\
\text { prefer in-person }(\mathrm{n}=347) \\
(\%)\end{array}$ \\
\hline Not at all or slightly & 15.4 & 32.3 \\
Moderately & 28.2 & 47.0 \\
Very or extremely & 56.4 & 20.7 \\
\hline
\end{tabular}

$N=386, \chi^{2}(2)=24.32, p<0.001$ 
were not first-time mothers. Given the small cell sizes among those who indicated "no preference," "somewhat prefer virtual prenatal care" or "very much prefer virtual prenatal care," we could only compare the proportion of first-time moms to those who had pregnancies before among those who indicated "very much prefer in-person" and "somewhat prefer in-person." We observed a statistical trend for mothers who were first time moms to be more likely to indicate "very much prefer in-person prenatal care" $(74.1 \%)$ than moms who were pregnant before $(65.2 \%)\left[\chi^{2}(1)=3.36, p=0.07\right]$.

\section{Discussion}

The COVID-19 pandemic has led to the experience of a sudden shift to virtual prenatal care for many women, representing a novel transformation for patient experience. The primary goal of our study was to understand the level of patient satisfaction on the virtual prenatal care experienced by women living in the U.S. during the COVID-19 pandemic. A second goal was to understand how mental health and emotional experiences relate to patient satisfaction with virtual prenatal care. Finally, we examined participant preferences for virtual care under non-pandemic conditions.

Our findings demonstrate general satisfaction with virtual prenatal care during the COVID-19 pandemic with $27.9 \%$ of our respondents either very or extremely satisfied with their virtual prenatal care experiences, and an additional $43.5 \%$ moderately satisfied. The high rates of satisfaction may be attributable to a shared desire by organizations and patients for minimizing in-person visits because of the pandemic (Jeganathan et al., 2020). These findings point to virtual care as a viable option during this public health crisis.

However, our data indicate an important caveat when considering virtual prenatal care beyond the pandemic. Notably, a large majority of women in our study (89.9\%) indicated a preference for in-person prenatal care under non-pandemic conditions. While women in our study were generally satisfied with their virtual prenatal care experiences during the pandemic, on average, they would have preferred in-person care if it were not for a pandemic. In other words, patient satisfaction of virtual care did not equate to patient preference for virtual care. Women may prefer an in-person visit because it provides a more ideal setting to get to know their provider or that it allows patient concerns to be shared in a way that cannot be done remotely. Such a preference may be attributed to a desire for a fuller physical exam experience (routine blood pressure measurement, detecting fetal heartbeat), that may be limited by a virtual prenatal care arrangement. That being the case, in a survey of patients before the pandemic, only $24 \%$ of patients expressed a preference for telemedicine for their prenatal care, even though most indicated being comfortable with remote monitoring (e.g., monitoring of blood pressure or fetal heartbeat at home) with the provision of the required equipment (Peahl et al., 2020a, 2020b).

Our data also showed that COVID-19 pregnancy worries and pandemic duration were associated with lowered satisfaction with virtual prenatal care (generalized anxiety symptoms were associated with lower satisfaction as well, but did not reach a statistical significance of $p<0.05$ ). The associations appear to be consistent with prior literature showing a link between psychological distress and lowered patient satisfaction (Boinon et al., 2018; Jibodh et al., 2010). State anxiety has been associated with lower levels of satisfaction with doctor's interpersonal skills, availability, and waiting time (Boinon et al., 2018). Those who are anxious or worried are less likely to have their expectations for care fulfilled. In fact, those with health worries have been shown to focus on negative and threatening information (Kaur et al., 2011, 2013); such attentional bias and the desire to seek reassurance from providers regarding their physical health, may not be readily achieved, particularly within a virtual format (Imlach et al., 2020). Health anxiety, that is, the interpretation of benign physical sensations as a sign of a disease, has been found to be associated with lower patient satisfaction (Tanis et al., 2016). Those who are pregnant women may be prone to health anxiety as it can be difficult to distinguish physical sensations that are typical of pregnancy from other concerns (Rathbone \& Prescott, 2019).

Unlike the worries pertaining to COVID-19 in pregnancy, worries about health related to COVID-19 (e.g., contraction of COVID-19) was not associated with patient satisfaction. Thus, the worries concerning COVID-19 on the pregnancy may be more salient than general health worries about COVID-19, when specifically considering patient satisfaction about prenatal care. Although COVID-19-related grief might be thought to affect one's satisfaction for virtual prenatal care in different ways (e.g., having less time or interest in attending in person visits), we did not see such experiences of grief related to their report of satisfaction with virtual prenatal care. Based on our data, it appears that concurrent distress about their pregnancy is likely more predictive of patient satisfaction.

The number of prenatal care changes due to the COVID19 pandemic contributed to lower satisfaction, even after accounting from the other factors. For the majority of respondents, the transition from in-person to virtual prenatal care visits, the format of their prenatal care, the cancellation or reduction in the frequency of prenatal visits, and a cancellation of hospital tours were among those changes. Continuity of care, that is, an "uninterrupted experience across the same hospital, location, or provider" (Batbaatar et al., 2017; Ware et al., 1977) is a consistent predictor of patient satisfaction in studies under non-pandemic conditions (Batbaatar et al., 2017). While the transition to virtual care may be 
understandable and necessary due to the pandemic, changes to the regular and expected routine appear to account for reported lower levels of satisfaction of virtual care among women in our study.

Interestingly, respondents who completed the survey further into the pandemic were less satisfied with their virtual prenatal care. This might reflect an expectation for higher quality of virtual prenatal care with the progression of time, whereas patients may have been more accommodating with the transition to virtual care in the earlier phases of the pandemic. This finding could also represent the expectation that a transition should be made back to in-person visits, with pandemic fatigue leading to individuals resuming regular routines (World Health Organization, 2020). Future work is needed to understand how patient satisfaction varies throughout the duration of an ongoing major event.

\section{Limitations}

We acknowledge the following limitations. The number of visits, the nature of the virtual experience (video or audio only, remote monitoring by the patient using a Doppler or blood pressure cuff on their own) (Pflugeisen \& Mou, 2017), and any hybrid configurations (virtual and in-person experiences) were not available, however we would presume that these varied experiences as well as provider characteristics might play a role in patient satisfaction. We relied on oneitem to assess patient satisfaction rather than a standardized measure; thus, our findings can only speak to the general experience rather than various domains of patient satisfaction (e.g., satisfaction regarding information, provider care, system characteristics) (Omar et al., 2001; Westaway et al., 2003). Although our study focused on patient satisfaction, cost effectiveness and quality of care delivery should also be considered when evaluating outcomes (Fryer et al., 2020).

We also note limitations in generalizability due to the variations in COVID-19 cases and hospitalizations based on location which may affect one's satisfaction or preference over virtual prenatal care. Given that we relied on an internet recruitment strategy and an online survey, a strength is our large sample as well as the ability to generalize our satisfaction and preference for virtual prenatal care findings to those with internet access who would be most likely able to take part in a virtual prenatal care experience. However, a major downside to our online convenience sampling design is that it disallows us to ascertain a response rate (limiting external validity) and it produces a skewed demographic of participants. Although internet recruitment may be perceived as far-reaching, there was a disproportionate rate of women in our sample with higher socioeconomic levels, higher levels of education, and who were White (Pew Research Center, 2021). Despite our efforts to recruit a diverse sample, our sample resulted in few Black (0.7\%) and Hispanic/Latina
(3.4\%) participants. Greater resources are needed to ensure the representation of these women in online studies in general. As well, future work should seek to understand their ability to take part in virtual prenatal care, and their satisfaction and preference for such a format.

Finally, causal inferences between our predictors and patient satisfaction cannot be determined due to the correlational nature of this data, and these associations cannot be generalized outside of the study sample.

Despite these limitations, the strengths of our study include a unique assessment of a cohort that was pregnant during the pandemic, and the inclusion of psychosocial experiences-variables that are often overlooked in studies on patient satisfaction. Together, these findings point to psychological experiences and emerging concerns relevant to patient satisfaction.

\section{Clinical Implications}

Providers and clinical settings should be aware that the number of changes involved in the prenatal care experience, including the transition from in-person to virtual care, the cancellation and or reduction of care, and the changes in the birth plan may play a major role in the patient satisfaction of virtual prenatal care. Streamlining the logistics involved in any changes and clear communication to patients may be key to ensuring patient satisfaction of their experience.

Given that pregnancy worries specific to the COVID19 pandemic may play a role in satisfaction, providers may consider discussing with patients the extent to which a virtual prenatal care arrangement is a safe and alternative option for in-person visits, and how fetal monitoring will be maintained through in-person visits or remote monitoring equipment (blood pressure monitors, fetal heart monitoring, tape measures) should such monitoring take place virtually. Recent study findings suggest the accuracy, feasibility, and acceptability of home-based wireless fetal heart (FHR) monitoring systems among low and high-risk pregnant women (Heuvel et al., 2020; Porter et al., 2021), with evidence indicating home-based FHR readings to be equivalent to those obtained in the clinic setting (Porter et al., 2021). Moreover, there is data to suggest that most pregnant women are satisfied with home-based FHR monitoring (Heuvel et al., 2020). However, the consideration of such an arrangement should be fully discussed with the patient.

Finally, psychosocial experiences including worry and anxiety may be linked to patient satisfaction. Healthcare providers may consider attending to the concerns of women worried about the impacts of the COVID-19 pandemic during pregnancy (Liu \& Doan, 2020; Liu et al., 2021b, c). Conversations about mental health within the prenatal care setting remains critical (Liu \& Tronick, 2012), as these are 
factors that might negatively affect women's perception of virtual prenatal care and provider-patient engagement.

Our data also suggests that despite the reported general satisfaction of virtual prenatal care, the large majority of women had a preference for in-person visits. Hospitals have had to make an abrupt transition to virtual care models due to the pandemic. The consideration of virtual components as a standard of care should not be abandoned as prenatal care clinics continue to evaluate such an option. Based on our results, it is necessary to carefully consider the design of a virtual or hybrid model through continual monitoring and improvements to ensure its success. Additional studies will be needed to understand the unmet needs through virtual or hybrid models compared to in-person standard of care.

Acknowledgements Support for this manuscript was provided through the Mary A. Tynan Faculty Fellowship, the Weinberg and Barton families, the Family Health and Resilience Fund, and a NIH K23 MH 107714-01 A1 award (to C.H.L.). We are grateful for the assistance of Sunah Hyun, Emily Zhang, and Ga Tin Finneas Wong in the collection of this data and for Ga Tin Finneas Wong in assisting with the preparation of this manuscript. This work has not been presented in any meetings nor has it been posted on a preprint server.

Author Contributions CHL and DG conceptualized the research question, analyzed the data, and wrote the manuscript. LM and CE assisted with the interpretation of findings and editing of the manuscript.

Funding Support for this manuscript was provided through the Mary A. Tynan Faculty Fellowship, the Weinberg and Barton Families, Family Health and Resiliency Research Fund, and a NIH K23 MH 10771401 A1 award (to C.H.L.).

\section{Declarations}

Conflict of interest The authors have no disclosures to declare.

Consent to Participate Participants consented to participation prior to the data collection, according to regulations set by the Institutional Review Board.

Consent to Publish No individual's data or image is presented in this paper.

\section{References}

Aziz, A., Zork, N., Aubey, J. J., Baptiste, C. D., D'alton, M. E., Emeruwa, U. N., Fuchs, K. M., Goffman, D., Gyamfi-Bannerman, C., \& Haythe, J. H. (2020). Telehealth for high-risk pregnancies in the setting of the COVID-19 pandemic. American Journal of Perinatology, 37(8), 800.

Batbaatar, E., Dorjdagva, J., Luvsannyam, A., Savino, M. M., \& Amenta, P. (2017). Determinants of patient satisfaction: A systematic review. Perspectives in Public Health, 137(2), 89-101.

Boinon, D., Dauchy, S., Charles, C., Fasse, L., Cano, A., Balleyguier, C., Mazouni, C., Caron, H., Vielh, P., \& Delaloge, S. (2018). Patient satisfaction with a rapid diagnosis of suspicious breast lesions: Association with distress and anxiety. The Breast Journal, 24(2), 154-160.

Fryer, K., Delgado, A., Foti, T., Reid, C. N., \& Marshall, J. (2020). Implementation of obstetric telehealth during COVID-19 and beyond. Maternal and Child Health Journal, 24(9), 1104-1110.

Futterman, I., Rosenfeld, E., Toaff, M., Boucher, T., Golden-Espinal, S., Evans, K., \& Clare, C. A. (2020). Addressing disparities in prenatal care via telehealth during COVID-19: Prenatal satisfaction survey in East Harlem. American Journal of Perinatology, $38,88-92$.

Holcomb, D., Faucher, M. A., Bouzid, J., Quint-Bouzid, M., Nelson, D. B., \& Duryea, E. (2020). Patient perspectives on audio-only virtual prenatal visits amidst the severe acute respiratory syndrome coronavirus 2 (SARS-CoV-2) pandemic. Obstetrics \& Gynecology, 136(2), 317-322.

Imlach, F., McKinlay, E., Middleton, L., Kennedy, J., Pledger, M., Russell, L., Churchward, M., Cumming, J., \& McBride-Henry, K. (2020). Telehealth consultations in general practice during a pandemic lockdown: Survey and interviews on patient experiences and preferences. BMC Family Practice, 21(1), 269. https://doi.org/10.1186/s12875-020-01336-1

Jeganathan, S., Prasannan, L., Blitz, M. J., Vohra, N., Rochelson, B., \& Meirowitz, N. (2020). Adherence and acceptability of telehealth appointments for high-risk obstetrical patients during the coronavirus disease 2019 pandemic. American Journal of Obstetrics \& Gynecology MFM, 2(4), 100233.

Jibodh, S. R., Kandil, A. O., Malchau, H., \& Estok, D. M., II. (2010). Do commonly reported outcome measures reflect patient satisfaction after revision hip arthroplasty? The Journal of Arthroplasty, 25(1), 41-45.

Kaur, A., Butow, P. N., \& Sharpe, L. (2013). Health threat increases attentional bias for negative stimuli. Journal of Behavior Therapy and Experimental Psychiatry, 44(4), 469-476. https://doi. org/10.1016/j.jbtep.2013.06.003

Kaur, A., Butow, P., \& Thewes, B. (2011). Do metacognitions predict attentional bias in health anxiety? Cognitive Therapy and Research, 35(6), 575-580. https://doi.org/10.1007/ s10608-011-9387-6

Kruse, C. S., Krowski, N., Rodriguez, B., Tran, L., Vela, J., \& Brooks, M. (2017). Telehealth and patient satisfaction: A systematic review and narrative analysis. British Medical Journal Open, 7(8), e016242.

Lewinsohn, P. M., Seeley, J. R., Roberts, R. E., \& Allen, N. B. (1997). Center for epidemiologic studies depression scale (CESD) as a screening instrument for depression among communityresiding older adults. Psychology and Aging, 12(2), 277-287. https://doi.org/10.1037//0882-7974.12.2.277

Liu, C. H., \& Doan, S. N. (2020). Psychosocial stress contagion in children and families during the COVID-19 pandemic. Clinical Pediatrics, 59(9-10), 853-855. https://doi.org/10.1177/00099 22820927044

Liu, C. H., Erdei, C., \& Mittal, L. (2021a). Risk factors for depression, anxiety, and PTSD symptoms in perinatal women during the COVID-19 Pandemic. Psychiatry Research, 295, 113552. https://doi.org/10.1016/j.psychres.2020.113552

Liu, C. H., Mittal, L., \& Erdei., C. (2021b). COVID-19-related health worries compound the psychiatric distress experienced by families of high-risk infants. Journal of Perinatology, 41(5), 1191-1195. https://doi.org/10.1038/s41372-021-01000-1

Liu, C. H., Smiley, P. A., Vicman, J. M., Wong, G. T. F., \& Doan, S. N. (2021c). The roles of life stress and preventive health behaviors on parent mental health during the COVID-19 pandemic. Journal of Health Psychology. https://doi.org/10.1177/13591053211026742

Liu, C. H., \& Tronick, E. (2012). Do patient characteristics, prenatal care setting, and method of payment matter when it comes to provider-patient conversations on perinatal mood? Maternal 
and Child Health Journal, 16(5), 1102-1112. https://doi.org/ 10.1007/s10995-011-0835-4

Lowery, C., DeNicola, N., \& American College of Obstetricians and Gynecologists' Presidential task force on telehealth. (2020). Implementing Telehealth in Practice (No. 798; Committee Opinion). American College of Obstetricians and Gynecologists. https://www.acog.org/en/Clinical/ClinicalGuidance/ Committee Opinion/Articles/2020/02/Implementing Telehealth in Practice

Merikangas, K., Milham, M., \& Stringaris, A. (2020). The Coronavirus health impact survey (CRISIS). Retrieved from http://www.crisi ssurvey.org/

National Center for Health Statistics. (2020). Provisional estimates for selected maternal and infant outcomes by month, 2018-2020. COVID-19 birth data and resources. Retrieved from https://www. cdc.gov/nchs/covid19/technical-notes-outcomes.html

Omar, M. A., Schiffman, R. F., \& Bingham, C. R. (2001). Development and testing of the patient expectations and satisfaction with prenatal care instrument. Research in Nursing \& Health, 24(3), 218-229.

Peahl, A. F., Powell, A., Berlin, H., Smith, R. D., Krans, E., Waljee, J., Dalton, V. K., Heisler, M., \& Moniz, M. H. (2020b). Patient and provider perspectives of a new prenatal care model introduced in response to the coronavirus disease 2019 pandemic. American Journal of Obstetrics and Gynecology, 224, 384.

Peahl, A. F., Novara, A., Heisler, M., Dalton, V. K., Moniz, M. H., \& Smith, R. D. (2020a). Patient preferences for prenatal and postpartum care delivery: A survey of postpartum women. Obstetrics \& Gynecology, 135(5), 1038-1046.

Pew Research Center. (2021). Demographics of internet and home broadband usage in the United States (internet \& technology). Pew Research Center.

Pflugeisen, B. M., \& Mou, J. (2017). Patient satisfaction with virtual obstetric care. Maternal and Child Health Journal, 21(7), 15441551. https://doi.org/10.1007/s10995-017-2284-1

Plummer, F., Manea, L., Trepel, D., \& McMillan, D. (2016). Screening for anxiety disorders with the GAD-7 and GAD-2: A systematic review and diagnostic metaanalysis. General Hospital Psychiatry, 39, 24-31. https://doi.org/10.1016/j.genhosppsych.2015.11.005

Porter, P., Muirhead, F., Brisbane, J., Schneider, B., Choveaux, J., Bear, N., Carson, J., Jones, K., Silva, D., \& Neppe, C. (2021). Accuracy, clinical utility, and usability of a wireless self-guided fetal heart rate monitor. Obstetrics and Gynecology, 137(4), 673-681. https://doi.org/10.1097/AOG.0000000000004322

Prigerson, H. G., Maciejewski, P. K., Reynolds, C. F., Bierhals, A. J., Newsom, J. T., Fasiczka, A., Frank, E., Doman, J., \& Miller, M. (1995). Inventory of complicated grief: A scale to measure maladaptive symptoms of loss. Psychiatry Research, 59(1-2), 65-79. https://doi.org/10.1016/0165-1781(95)02757-2

Radloff, L. S. (1977). The CES-D scale: A self-report depression scale for research in the general population. Applied Psychological
Measurement, 1(3), 385-401. https://doi.org/10.1177/0146621677 00100306

Rathbone, A. L., \& Prescott, J. (2019). Pregnancy-specific health anxiety: Symptom or diagnosis? British Journal of Midwifery, 27(5), 288-293.

Reforma, L. G., Duffy, C., Collier, A.-R.Y., Wylie, B. J., Shainker, S. A., Golen, T. H., Herlihy, M., Lydeard, A., \& Zera, C. A. (2020). A multidisciplinary telemedicine model for management of coronavirus disease 2019 (COVID-19) in obstetrical patients. American Journal of Obstetrics \& Gynecology MFM, 2(4), 100180. https://doi.org/10.1016/j.ajogmf.2020.100180

Spitzer, R. L., Kroenke, K., Williams, J. B., \& Löwe, B. (2006). A brief measure for assessing generalized anxiety disorder: The GAD-7. Archives of Internal Medicine, 166(10), 1092-1097. https://doi. org/10.1001/archinte.166.10.1092

Tanis, M., Hartmann, T., \& Te Poel, F. (2016). Online health anxiety and consultation satisfaction: A quantitative exploratory study on their relations. Patient Education and Counseling, 99(7), 1227-1232.

van den Heuvel, J. F. M., Teunis, C. J., Franx, A., Crombag, N. M. T. H., \& Bekker, M. N. (2020). Home-based telemonitoring versus hospital admission in high risk pregnancies: A qualitative study on women's experiences. BMC Pregnancy and Childbirth, 20(1), 77. https://doi.org/10.1186/s12884-020-2779-4

Ware, J. E., Davies, A. R., \& Stewart, A. (1977). The measurement and meaning of patient satisfaction: A review of the literature. RAND Corporation.

Weathers, F. W., Litz, B. T., Herman, D. S., Huska, J. A., \& Keane, T. M. (1993). The PTSD checklist (PCL): Reliability, validity, and diagnostic utility. Annual Convention of the International Society for Traumatic Stress Studies, San Antonio, TX, 462

Westaway, M. S., Rheeder, P., Van Zyl, D. G., \& Seager, J. R. (2003). Interpersonal and organizational dimensions of patient satisfaction: The moderating effects of health status. International Journal for Quality in Health Care, 15(4), 337-344.

World Health Organization. (2020). Pandemic fatigue: Reinvigorating the public to prevent COVID-19: policy considerations for Member States in the WHO European Region (WHO/EURO:20201160-40906-55390). World Health Organization. Regional Office for Europe. Retrieved from https://apps.who.int/iris/handle/10665/ 335820

Zork, N. M., Aubey, J., \& Yates, H. (2020). Conversion and optimization of telehealth in obstetric care during the COVID-19 pandemic. Seminars in Perinatology, 44(6), 151300.

Publisher's Note Springer Nature remains neutral with regard to jurisdictional claims in published maps and institutional affiliations. 